\title{
PRIMER REGISTRO DE MYMAR TAPROBANICUS WARD, 1875 (HYMENOPTERA: CHALCIDOIDEA: MYMARIDAE) PARA LA REPÚBLICA DOMINICANA
}

\author{
Santo Navarro Morales \\ Instituto de Investigaciones Botánicas y Zoológicas Prof. Rafael Ma. Moscoso, Universidad Autónoma \\ de Santo Domingo.n.santo9@gmail.com
}

\section{RESUMEN}

Se cita por primera vez para la República Dominicana la avispa parasítica Mymar taprobanicus Ward, 1875. Este registro se basa en un espécimen macho colectado en la Reserva Científica Loma Barbacoa, provincia Peravia.

Palabras clave: Mymar, Mymaridae, Chalcidoidea, Loma Barbacoa, República Dominicana, La Española, Las Antillas.

Title: First record of Mymar trapobanicus Ward, 1875 (Hymenoptera: Chalcidoidea: Mymaridae) for the Dominican Republic.

\section{ABSTRACT}

The parasitic wasp Mymar taprobanicus Ward, 1875 is reported for the first time to the Dominican Republic. This report is based on one male specimen collected in the Loma Barbacoa Scientific Reserve, Peravia province.

Keywords: Mymar, Mymaridae, Chalcidoidea, Loma Barbacoa, Dominican Republic, Hispaniola, West Indies.

Los miembros de la familia Mymaridae son himenópteros de distribución mundial y comprenden los insectos más pequeños del orden (Goulet y Huber, 1993; Fernández y Sharkey, 2006; Lin et al., 2007). Estos son parásitos de huevos de hemípteros, homópteros, lepidópteros y dípteros (Alayo y Hernández, 1978; Yoshimoto, 1984; Guzman-Larralde, 2001). De los mimáridos se conocen aproximadamente 100 géneros y 1400 especies en el mundo (Loiácono et al., 2012; Noyes, 2012). Los individuos pertenecientes a este grupo se caracterizan por ser diminutos, de colores no metálicos, con gran reducción de la venación en las alas anteriores y variación en el tamaño, oscilando esta última entre 0.2 y $4 \mathrm{~mm}$ (Yoshimoto, 1990). A pesar de su abundancia e importancia económica, por su papel como biorreguladores, los mimáridos son un grupo escasamente conocido. El género Mymar es de distribución mundial e incluye once (11) especies (Noyes, 2012). En el Caribe, M. taprobanicum ha sido citada para Puerto Rico (como Mymar antillanum Dozier, 1937) y para muchos países en todos los continentes (Noyes , 2012; Annecke, 1961; Beardsley y Huber, 2000). Este es el segundo registro del género para el Caribe insular y el primer registro para La Española.

En la lista de artrópodos de la Hispaniola (Perez-Gelabert, 2008) se incluyen siete géneros y ocho especies de Mymáridos. Para la determinación de los géneros de Mymaridae, se han desarrollado diversas claves, entre las que se destacan tanto la de Debauche (1949) como la de Anneke y Doutt (1961). Para la identificación a nivel de géneros del mundo, Yoshimoto (1990), para identificar los géneros del nuevo mundo, aunque en su mayoría no están actualizadas (Lin et al., 2007). Para la determinación de nuestro ejemplar, usamos a Yoshimoto (1990). 

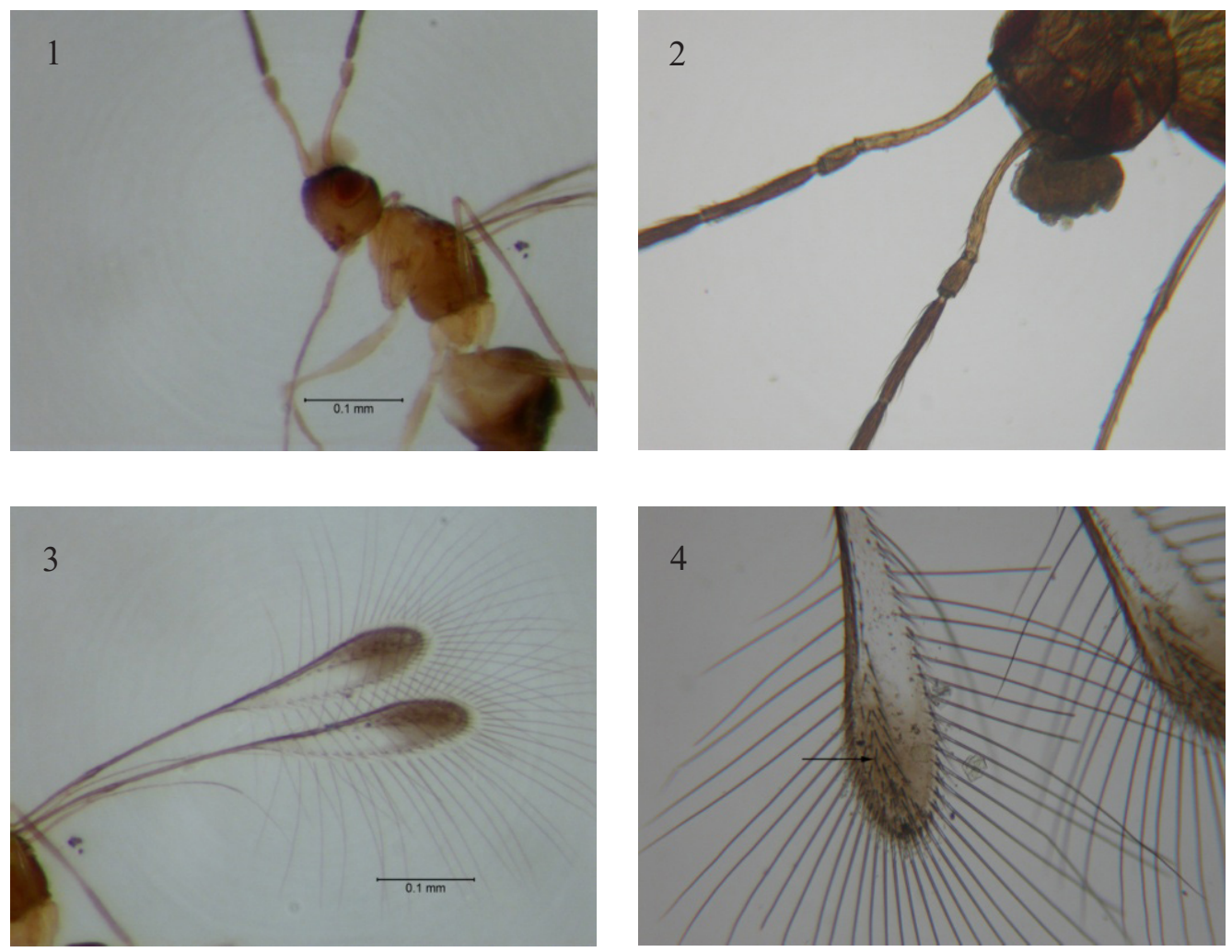

Figuras 1-4. Macho Mymar taprobanicus. 1. Vista ventrolateral mostrando la coloración típica de este grupo. 2. Cabeza y antena mostrando detalles de escapo y el pedicelo. 3. Vista del ala anterior. 4. Acercamiento mostrando el penacho de setas y varias líneas de setas que inician desde la parte media de la expansión alar (flecha).

Diagnosis del macho. El ejemplar examinado mide menos de $1 \mathrm{~mm}$ y presenta una coloración amarillo naranja sin brillo metálico. Las antenas presentan trece segmentos, siendo el escapo 3.2 veces más largo que el pedicelo (Fig. 2). Las alas anteriores pedunculadas ensanchándose en el último tercio y terminando con un penacho de flecos (Fig. 3). Varias líneas diagonales de cortas setas marcan el área terminal membranosa de estas alas (Fig. 4). La parte central de la última porción del ala es hialina. Avispa de color amarillo sin brillo metálico.

Antena. La antena es muy larga y filiforme, con 13 segmentos. El escapo algo curvo, casi cinco veces más largo que ancho; el pedicelo algo englobado y al menos 2.5 veces menor que el primer segmento funicular, todos los segmentos funiculares aproximadamente del mismo tamaño y grosor, cubiertos de una fina pilosidad.

Alas. Las alas anteriores pedunculadas a manera de remos y con un penacho de largos flecos al final; total de flecos: 42; alas posteriores atrofiadas, mayormente filiformes, desnudas en los primeros dos tercios, último tercio con varias hileras de pequeñas setas, tórulos antenales ampliamente separados.

Cabeza. Es ancha, con ojos ampliamente separados, grandes y de color rojo; las antenas ubicadas en la parte superior de la cabeza. Distancia antena ojo (AO) más corta que la distancia entre ellas. Propodeum y la mitad del metasoma de color amarillo pálido, la segunda mitad de este 
último de color marrón oscuro (Fig. 1), antenas y patas castaño oscuro. Coxas globulares, siendo las procoxas ligeramente más grandes que las meso y metacoxas. Tarsos con cuatro segmentos, el primer segmento casi tan largo como el segundo y el tercero juntos; metasoma peciolado y con coloración amarillo claro.

Material estudiado. Un único individuo macho, colectado a 1361 metros sobre el nivel del mar (msnm) en la Reserva Científica Loma Barbacoa, Prov. Peravia. Fecha de colecta: 30/IX al 4/X2011. El ejemplar está depositado en la colección entomológica del Instituto de Investigaciones Botánicas y Zoológicas Prof. Rafael Ma. Moscoso (IIBZ) de la Universidad Autónoma de Santo Domingo (UASD).

\section{AGRADECIMIENTOS}

Este trabajo ha sido posible gracias al patrocinio del Ministerio de Educación Superior Ciencia y Tecnología a través del Proyecto FONDOCYT No. 2009-102. Los Doctores Daniel E. Pérez-Gelabert y Julio A. Genaro revisaron el manuscrito e hicieron importantes sugerencias. Un agradecimiento muy especial a Candy Ramírez y Fritz Pichardo Marcano por las fotografías de la avispa.

\section{LITERATURA CITADA}

Alayo D. y L. R. Hernández. 1978. Introducción al estudio de los himenópteros de Cuba: Superfamilia Chalcidoidea. Academia de Ciencias de Cuba, La Habana, 105 pp.

Annecke, D. P., y R. L. Doutt. 1961. The genera of the Mymaridae (Hymenoptera: Chalcidoidea). Republic of South Africa, Department of Agricultural Technical Services, Entomology Memoirs, vol. 5, 71 pp.

Beardsley J. W. y J. T. Huber. 2000. Key to genera of Mymaridae in the Hawaiian Islands, with notes on some of the species (Hymenoptera: Chalcidoidea). Proceedings of the Hawaiian Entomological Society, 34: 1-22.

Dozier, H. L. 1937. Description of miscellaneous chalcidoid parasites from Puerto Rico. (Hymenoprtera). The Journal of Agriculture of the University of Puerto Rico, 21: 121-135.

Fernández, F. y M. J. Sharkey (eds.). 2006. Introducción a los Hymenoptera de la Región Neotropical. Sociedad Colombiana de Entomología y Universidad Nacional de Colombia, Bogotá D. C., xxx +894 pp.

Goulet, H. y J. T. Huber, 1993. Hymenoptera of the World: an identification guide to families, Centre for Land and Biological Resources Research, Ottawa, Ontario, 668 pp.

Guzman-Larralde, A. J., J. L. Leyva y J. M. Valdez. 2001. Illustrated key to genera of Mymaridae (Hymenoptera) of central Mexico. Southwestern Entomologist, 26: 245-252.

Lin, N.-Q., J. T. Huber y J. La Salle, 2007. The Australian genera of Mymaridae (Hymenoptera: Chalcidoidea). Zootaxa, 1596: 1-111.

Loiácono M. S., N. B. Díaz y L. De Santis. 2002. Estado actual del conocimiento de Microhimenópteros Chalcidoidea, Cynipoidea y "Proctotrupoidea" en Argentina. III. Marco sistemático del proyecto Pribes 2002: 221-230. 
Noyes, J. S. 2012. Universal Chalcidoidea Database. World Wide Web electronic publication. http://www.nhm.ac.uk/chalcidoids. Accesado en Octubre 23, 2012.

Perez-Gelabert, D. E. 2008. Arthropods of Hispaniola (Dominican Republic and Haiti): A checklist and bibliography. Zootaxa, 1831: 1-530.

Transactions of the Linnean Society of London, $2^{\text {nd }}$ series, 1: 583-593.

Yoshimoto, C. M. 1984. The Insects and Arachnids of Canada, part 12, Biosystematics Research Institute, Otawa, Ontario, 148 pp.

Westwood, J. O. 1879. Descriptions of some minute hymenopterous insects.

[Recibido el 06 de junio del 2014. Aceptado para publicación el 26 de agosto del 2014]. 